\title{
Recall of scenes encoded from opposing viewpoints
}

\author{
Kaitlin M. Varner, Stephen Dopkins, Hyun K. Kim, and John W. Philbeck \\ The George Washington University, Washington, DC, USA
}

\begin{abstract}
The present research examined the effect, on recall of a scene, of the distribution of viewpoints during the encoding of the scene. Participants completed a visual search task in a 3-D model of a room during which they were given the opportunity to view the room from viewpoints at opposing sides of the room. Subsequently, participants were tasked to recall the locations of the objects in the room. Participants who distributed their views of the room unequally across available viewpoints remembered the objects in the room as being too close to their preferred side of the room. Participants who distributed their views of the room equally across available viewpoints remembered the objects in each half of the room as being too close to the corresponding side of the room. Mindful of previous research that has shown exocentric distances to be underestimated along the depth dimension (Loomis, DaSilva, Fujita, \& Fukusima, 1992; Wu, He, \& Ooi, 2008), we suggest that the present results reflect underestimation of the distance between the objects and the sides of the room. We suggest that participants used an accumulator-like process to integrate the inconsistent location information that they acquired consequent to theirunderestimation of distances from opposing viewpoints (Heathcote \& Love, 2012). Keywords: scene recall, distance perception, viewpoint.
\end{abstract}

Received 21 January 2014; received in revised form 03 July 2014; accepted 16 July 2014. Available online 25 November 2014.

\section{Intoduction}

Scene memory is necessary for a variety of everyday functions, including navigation and the guidance of action. Despite its ubiquitous nature, our understanding of the mechanisms underlying scene memory remains limited. Current research in this field typically expands upon findings from studies of object representation (Edelman, 1999). Conceptualization with respect to object and scene representation is often driven by the issue of reconciling changes in appearance to the same scene or object as a result of changes in viewpoint (Tarr, 1995; Friedman \& Waller, 2008; Mou, Zhang, \& McNamara, 2009; Ullman, 1998).

In order to examine how different views are integrated, studies of scene memory frequently follow a common paradigm. Participants are asked to study two views of the same scene, typically separated by approximately $20-30^{\circ}$ (Castelhano, Pollatsek, \& Rayner, 2009; Diwadkar \& McNamara, 1997; Friedman \& Waller, 2008). Following the study phase, a test phase presents either a new view of the same scene or a repeat of one of the views from the study phase. Participants are asked to assess whether or not the test view is new

Kaitlin M. Varner, Department of Psychology, The George Washington University; Stephen C. Dopkins, Department of Psychology, The George Washington University; John W. Philbeck, Department of Psychology, The George Washington University. Correspondence regarding this article should be directed to: Kaitlin M. Varner, Department of Psychology, The George Washington University, 2125 G St NW, Washington, DC 20052. Email: kmvarner@gwu.edu to them. Studies using this paradigm have yielded important insights on how mental representations are used to recognize scenes. However, the need to integrate views of a scene that diverge by more than 30 degrees is not at all uncommon. Little research has examined how individuals do this. In particular, little research has examined how participants integrate scenes that they come to know from opposing points of view. The present study addressed this question.

The study asked participants to demonstrate their stored representation of a scene that they acquired through a process of incidental learning. The stimulus was a model of a room created with the 3-D modeling software Google Sketchup (Figure 1). Participants created a mental representation of the room incidentally to searching for target objects (small cubes) during brief $(2 \mathrm{sec})$ views of the room. Participants were asked to direct their search by selecting a point of view prior to each attempt at locating a new target. To do this, participants chose one of two possible sides of the room ${ }^{1}$. Participants' point of view was then one of the two corners that made up the selected side, with the specific corner being randomly determined (it is important to note participants' decision was really between the two available sides, with the specific corner being chosen randomly). Sample images of these points of view can be seen in Figure 1.

${ }^{1}$ Viewpoint decisions were left up to participants because we were concerned, given that ours was an incidental learning paradigm, that forcing participants into a pattern of viewpoint choice that was not their natural choice might interfere with their ability to perceive and encode the object locations. 

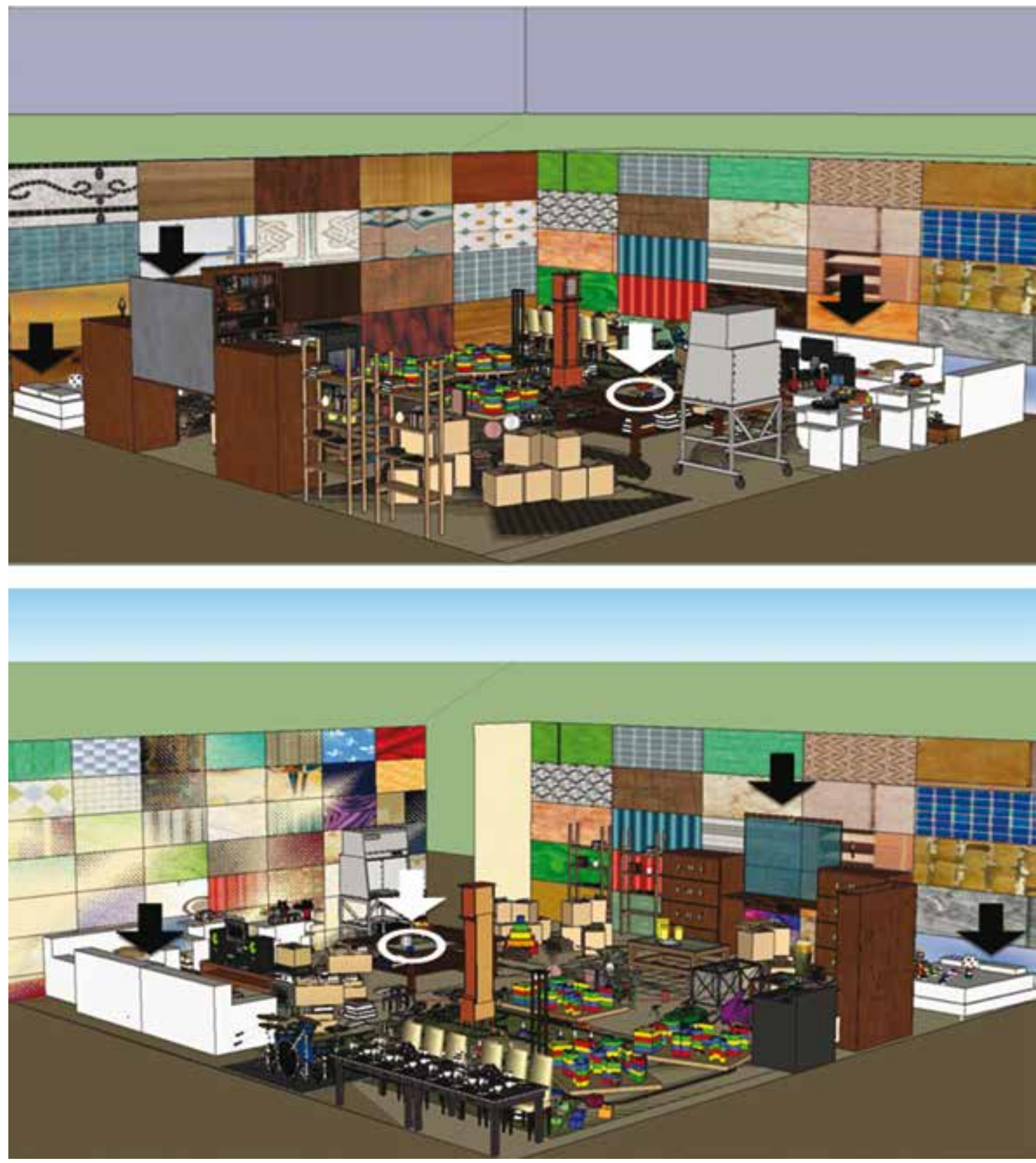

Figure 1. Sample image of the room to which participants were exposed during the search task. The top view corresponds to the corner labeled "A" in Figure 2; the bottom view corresponds to the corner labeled "C" in Figure 2. White arrows indicate the locations of the search task targets in these views. Black arrows indicate the objects corresponding to the landmark icons for reconstruction task.

After completing the search task, participants were asked to complete a reconstruction of the room seen during the search task. This reconstruction task was meant to capture how participants combined their different views of the room into one representation. Similar to previous spatial memory paradigms (Cherry \& Park, 1993; Laurance et al. 2002), participants were given a sheet with an outline indicating the walls of the room, along with icons representing the major objects in the room, and asked to place the icons in their correct locations. The selected objects were picked because they were viewable from all four corners; this multiplicity of views would presumably favor these objects in representations of the scene.
Previous research has shown that scene recognition is intrinsically connected to viewpoint information (Castelhano et al., 2009; Diwadkar \& McNamara, 1997; Friedman \& Waller, 2008; Friedman et al., 2011; Mou et al., 2009; Mou, Fan, McNamara, Owen; 2008). With this in mind, we expected to see a clear impact of participants' behavior during the search task, and by extension their different views of the room, on their recall of the scene. By examining the relationship between the manner in which participants viewed the room and their memory for the room, we sought to gain more detailed insight on how scene representations are formed from opposing viewpoints. Specifically, we sought to determine how participants' representations of 
the room varied as their experiences of the room were distributed more equivalently between the two opposing sides.

Although there is a variety of information and cues participants might use in constructing their scene representation, a basic element of any representation is an estimate of the distances present in the scene. Previous research has found that exocentric distance is incorrectly estimated along depth intervals (Gogel, 1965; Loomis, Da Silva, Fujita, \& Fukusima, 1992; Wu, He, \& Ooi, 2008). Because participants learn the room from its sides, we assume that distances from the sides to objects in the room will be incorrectly estimated. However, we do not know how this will affect the overall memory representation, given that participants will learn the room from opposing sides. For example, if object A's distance is estimated from side 1 and then subsequently estimated from the opposing side 2 , then combining the views from the two sides will require reconciliation of inconsistent estimates of object A's distance from the two sides. The need for such reconciliation will increase as views become more equally distributed across available viewpoints. This research seeks to examine how such inconsistencies are resolved.

\section{Methods}

\section{Participants}

Forty undergraduate students at The George Washington University received course credit for their participation. The age range was $18-22$ years.

\section{Apparatus}

The search task was completed in a model of a rectangular room created using the 3-D modeling software Google Sketchup Version 8 (dimensions of floor: $10.23 \mathrm{~m}$ by $9.94 \mathrm{~m}$, as arbitrarily defined by the modeling software). The floor of the room was gray and the walls were covered in abstract patterns (Figure 1). It was possible for a participant to view the room from any of the four corners (Figure 2). In order to make the interior view of the room visible from a given corner, the two walls that intersected to form the corner were cut away, while the two walls that intersected to form the diagonally opposite corner were left intact. Behind the remaining walls a "horizon" was presented to help provide depth information. Participants used a Dell OptiPlex GX270 with an Intel Pentium 4 processor (2.26 GHz, $512 \mathrm{MB}$ RAM), with a 17-inch monitor. The search task was presented using E-Prime Version 2.10.2. For the reconstruction task, participants were given a large sheet of paper, which contained a toscale representation, such that $24.98 \mathrm{~m}$ in the units of the computer-generated room was equivalent to $1 \mathrm{~m}$ on the reconstruction map. This resulted in $.41 \mathrm{~m} \times .4 \mathrm{~m}$ outline representing the floor plan of the room. In order to help orient participants, three landmark icons representing three larger objects, located near the sides of the room (Figure 1), were placed in their correct

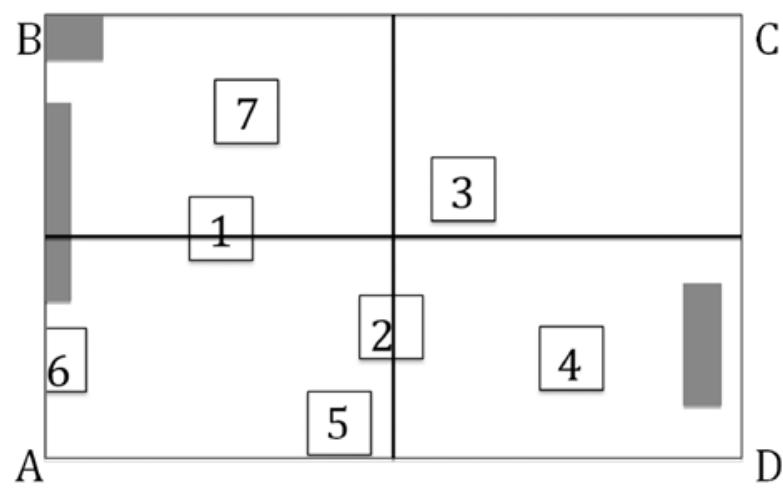

Figure 2. Schematic of the potential viewpoints during the search task and the object locations. If participants selected Side "1" during the search task, they were equally likely to view the room from the viewpoint labeled "A" and the viewpoint labeled "B". If participants selected Side "2" during the search task, they were equally likely to view the room from the viewpoint labeled " $\mathrm{C}$ " and the viewpoint labeled " $D$ ". Landmark icons presented during testing are shown as gray bars.

locations on the map. The bases of these icons were to-scale, with a range of sizes from $.13 \mathrm{~m} \times .03 \mathrm{~m}$ to $.17 \mathrm{~m} \times .02 \mathrm{~m}$ (Figure 2). To create their reconstruction, participants were given icons representing seven major objects from the room. The bases of these icons were toscale, with a range of sizes from $.02 \mathrm{~m} \times .01 \mathrm{~m}$ to $.07 \mathrm{~m}$ $\times .05 \mathrm{~m}$. All icons for this task represented objects that were highly visible during the search task, with minimal occlusion from all four possible viewpoints, although their appearance may have differed slightly depending on the specific viewpoint.

\section{Search task}

Participants were seated at a computer and began the search task by completing a practice trial. Before starting the practice trial participants were informed that they would experience a very brief $(2 \mathrm{~s})$ view of a computer generated room, and that their task was to locate a target (a small blue cube) within the room. After the view was completed, the participants were shown a longer duration $(5 \mathrm{~s})$ view of the domain with the target highlighted so that they could check their accuracy. Participants were then informed that the following trials would proceed in the same manner, with the targets always being cubes identical in shape and size to the cube seen during the practice trial, but with the targets being any one of four possible colors (blue, green, pink or purple). Participants were told that each view would contain exactly one target, that the targets could be hidden anywhere in the room and that their task on each trial would be to locate a target and subsequently report on the target's location.

At the beginning of each experimental trial, participants were asked to select a view of the room, out of two "side" options. "Side 1" was equally likely to present participants with a view from either corner A or corner B, whereas "Side 2" was equally likely to present participants with a view from either corner 
C or corner D (Figure 2). Notice that this procedure provided participants with the opportunity to see the room from all four corners but constrained choice to two options to minimize confusion about which view was being presented. Although participants saw the room from its four corners, their viewpoint decisions determined only the side from which they saw the room. More specifically, participants' viewpoint decisions resulted in their seeing the room from locations at opposing ends of the horizontal axis. We reasoned that, because participant decisions distributed responses between the two ends of the horizontal axis, the axis should be more salient to participants than the vertical axis (such a differentiation might also be fostered by the fact that the horizontal axis was longer than the vertical axis).

After selecting a side, participants were immediately presented with a brief $(2 \mathrm{~s})$ view of the room containing a target. Across the experiment, targets appeared in 60 different locations, distributed throughout the room. Targets were always in plain sight, with no occlusion by other objects but the short duration of the view and small size of the targets limited the participant's accuracy. Sample targets can be seen highlighted in white circles marked by white arrows in Figure 1. Following their view of the room, participants were presented with an image of the room that was covered with a grid structure (Figure
3) and instructed to input the number corresponding to the location of the target. Participants were given feedback on their accuracy for the current trial as well as a running total of their accumulated correct responses. The feedback did not demonstrate the correct location if the participants' response was incorrect. Immediately following their feedback, participants were returned to the start, and began a new trial by selecting a side. Participants were then presented with a new view of the room, containing a new target. Participants continued along these steps until they reached a total of 60 views of the room.

\section{Reconstruction task}

After completing their 60 views of the room, participants were asked to perform a reconstruction task. They were provided with the to-scale model of the room, as well as the seven to- scale icons representing seven objects from the room. The experimenter first placed the three landmark icons for participants, both to demonstrate the task and to provide cues to help orient participants (Figure 2). Participants were then instructed to place all seven icons into the model. In the event that participants did not recall seeing the object corresponding to a particular icon, the experimenter instructed them to make their best guess, and to not leave any objects out of the reconstruction.

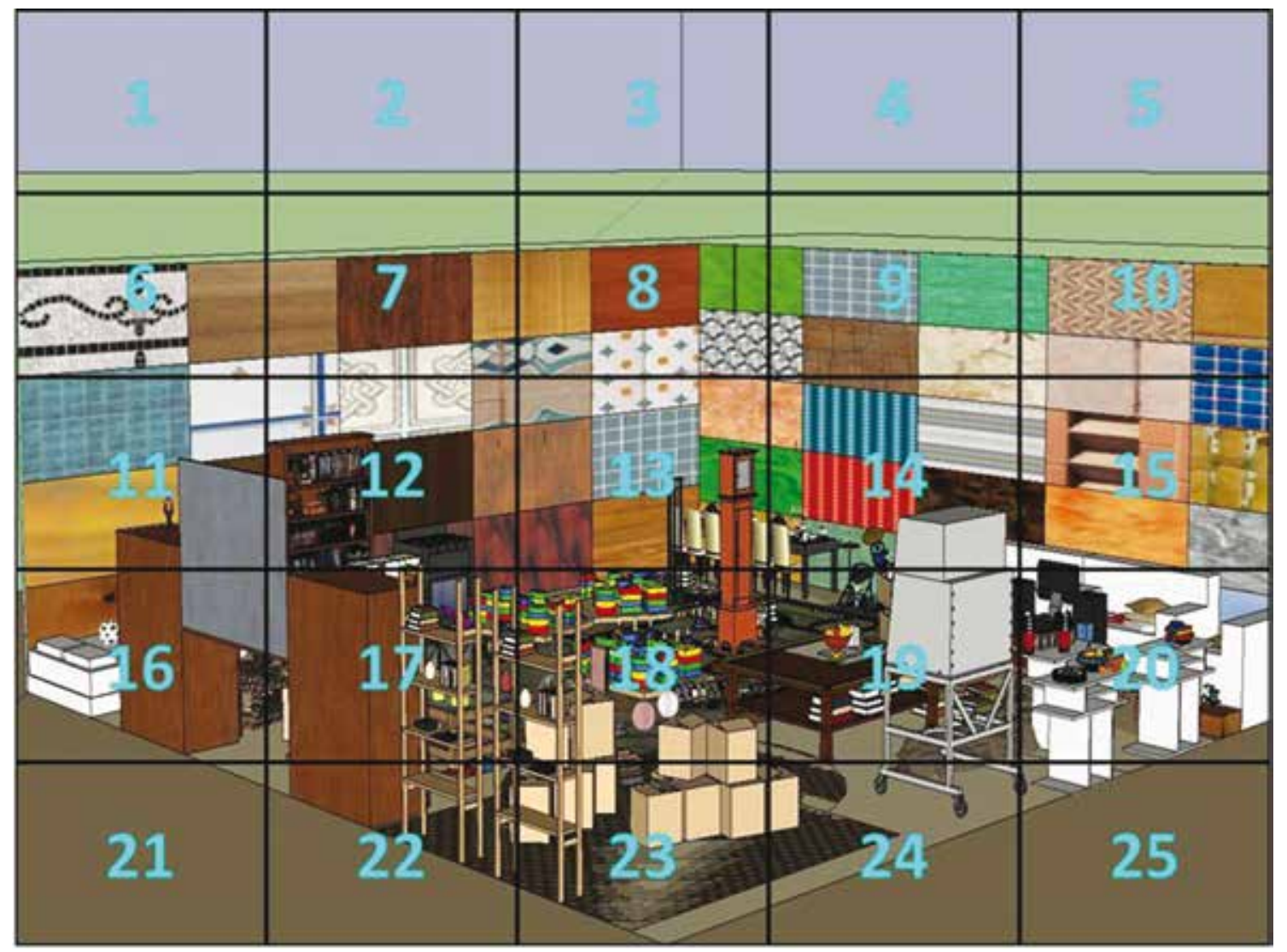

Figure 3. Sample image of response screen during search task. Participants were instructed to input the number of the grid that contained the target during the experimental view. 


\section{Results}

\section{Search task}

In order to index the distribution of viewpoints from which participants performed the search task, participants' selection of Side 1 was recorded as 1 , and participant's selection of Side 2 was recorded as 2 . The numbers corresponding to each participant's selections were then averaged and the absolute value of the difference between this average and 1.5 was calculated. This was done in order to collapse across sides, such that the absolute difference for each participant represented the participant's distance from equal distribution, regardless of the participant's specific side selections. That is, if a participant always chose Side 1 or Side 2, the participant's average side selection would be 1 or 2 and the participant's absolute difference would be 0.5 . If a participant chose Side 1 exactly half the time and Side 2 the other half, the participant's average side selection would be a 1.5 , and the associated absolute difference would be 0 . The distribution index for a given participant was then .5 minus the participant's absolute difference. Notice that, with this adjustment, the distribution index increased as distribution across available viewpoints approached equivalence. The mean of the distribution index across participants was significantly different from 0 , indicating a marked tendency on the part of participants to view the room from opposing points of view (Mean $=0.26, \mathrm{SE}=.02, \mathrm{t}$ $=11.26, \mathrm{p}<.0001$ ).

The proportions of times the two sides of the room were chosen were calculated for each participant. Participants did not demonstrate a significantly higher proportion for either side of the room (Mean of Side $1=$ $.47, \mathrm{SE}=.04, \mathrm{t}=-.28, \mathrm{p}>.05$ ).

Search task performance was evaluated as the proportion of hits out of the total attempts to provide a target location (Mean $=.48, \mathrm{SE}=.03$ ). The relationship between search task performance and participants' viewpoint distribution was examined. Search task performance decreased with increases in the distribution index $(\beta=-.64, \mathrm{t}=-2.51, \mathrm{p}=.02)$.

\section{Reconstruction task}

For both correct object locations and participants' responses, a center point was defined for the base of each object icon, using an average of the $\mathrm{x}$ and $\mathrm{y}$ coordinates of the corners of the base. Participants experienced some difficulty during the reconstruction task, as they were not warned ahead of time that they would need to complete it. As a result, overall error (i.e., unsigned distance between the correct object location and the participant's response location) was significantly different from zero (Mean $=.21 \mathrm{~m}, \mathrm{SE}=$ $.006 \mathrm{~m}, \mathrm{t}=-35.33, \mathrm{p}<.0001)$. Note that these units and all following units correspond to the 2-D reconstruction map, rather than the 3-D model. Overall error was not significantly predicted by the distribution index $(\beta=$ $24.99, \chi^{2}=.41, \mathrm{p}=.52$ ).

We next did an analysis to find the average direction of error for each object and across objects. To do this, we found the average error for each object (the centroid of the object's response placements when the correct object location was defined as 0,0 (Figure 4)). We then found the average error across objects (the centroid

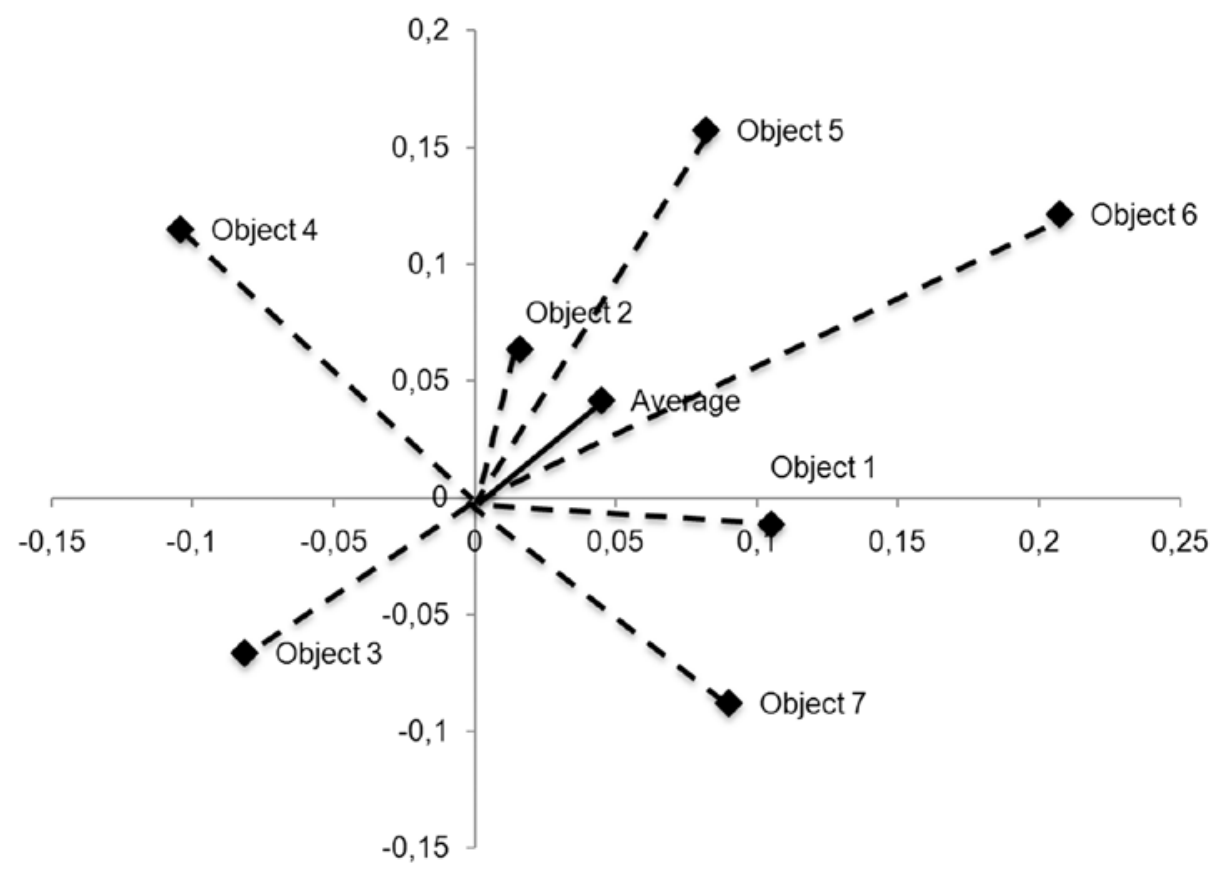

Figure 4. Average error vectors, in the horizontal and vertical direction, for test objects when the correct object location was defined as 0,0 . Vectors for test objects are shown with dashed lines. The average error vector (across all objects) is shown with the solid line. 
of the centroids). As can be seen in the figure, objects tended to be mislocated toward corner $\mathrm{C}$.

We then decomposed error in terms of polar coordinates in order to examine the relationship between object placements and the center of the room. This relationship can indicate whether objects are being clustered towards the center or spread out towards the four walls of the room. In the polar analyses, an object's radial coordinate was its Euclidean distance from the center of the room. The signed radial error for a given participant was the signed difference, across objects, between: (1) the correct distance between an object and the center of the room and (2) the participant's response distance between an object and the center of the room (Mean $=-.02 \mathrm{~m}, \mathrm{SE}=.003 \mathrm{~m}$ ). This negative mean indicates that participants' response distances tended to be larger than the correct distances between the objects and the center of the room, and by extension, that objects were spread out towards the sides of the room.

Our first attempt at assessing the correlational relationship between search task performance and memory for object location involved examining the participants' error in terms of distance from the center of the room. Using a generalized linear model we found no relationship between proportion of search task hits $\left(\beta=-28.11, \chi^{2}=2.16, p=.14\right)$ and signed radial error. There was a significant negative relationship between distribution index and signed radial error $(\beta=-50.63$, $\chi^{2}=5.8, \mathrm{p}=.02$ ) (Figure 5). As the distribution index increased, signed radial error became increasingly negative, indicating that participants placed the objects increasingly far from the center of the room.

To clarify the nature of the signed radial error effect, we examined whether the distribution index predicted the placement of objects as too far from vertical or horizontal midline. Given that participants viewed the room from corners A or B when they selected Side 1, and from corners $\mathrm{C}$ or D when they selected Side 2, and given that the two pairs of corners faced each other across the vertical midline, we hypothesized that the main effect of the distribution index would be on error in distance from the vertical midline (i.e., participants would place objects too far from the vertical midline). To test this hypothesis, we calculated each participant's signed error, across objects, in distance from the vertical and horizontal midlines. The signed error in distance from a given midline was the difference between the correct perpendicular distance from the midline and the participant's perpendicular response distance from the midline [Vertical Mean $=-.02 \mathrm{~m}, \mathrm{SE}=.003 \mathrm{~m}$; Horizontal Mean $=-.01 \mathrm{~m}, \mathrm{SE}=.003 \mathrm{~m}]$ (Figures 6-7). There was a significant negative relationship between the distribution index and signed error in distance from the vertical midline $\left(\beta=-54.98, \chi^{2}=8.01, p=.0047\right)$ (Figure 7). As the distribution index increased, signed error in distance from the vertical midline became increasingly negative, indicating that participants were placing objects further from the vertical midline. There was no correlational relationship between proportion of search task hits and signed error in distance from the vertical midline $\left(\beta=12.71, \chi^{2}=.44, p=.51\right)$.

Finally, no significant relationships were found between distribution index $\left(\beta=3.13, \chi^{2}=.03, p=.86\right)$ or proportion of search task hits $\left(\beta=-16.14, \chi^{2}=1.07\right.$, $\mathrm{p}=.3$ ) and signed error in distance from the horizontal midline.

Additionally, we compared the signed error in distance from the vertical midline for objects that were located in the halves of the room corresponding to participants' preferred and non-preferred sides of the room. Participants choosing Side 1 more than $60 \%$ of the time or less than $40 \%$ of the time were classified as preferring Sides 1 and 2, respectively (participants choosing Side 1 less than $60 \%$ of the time and more than $40 \%$ of the time were excluded as lacking distinct side preferences). The average signed error in distance from the vertical midline was then determined for objects in the halves of the room corresponding to each participant's preferred and non-preferred sides. Each object was assigned to a group based on its relationship to

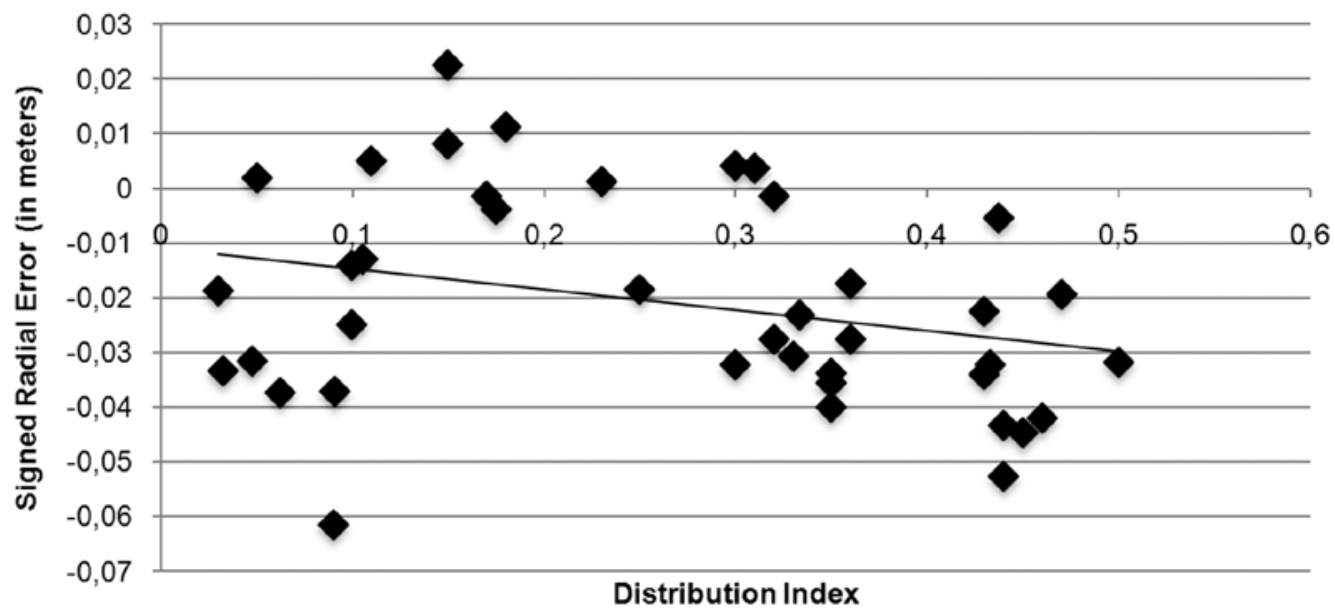

Figure 5. Relationship between distribution index and signed radial error. As the distribution index increased, signed radial error became increasingly negative, showing that on average objects were placed further than they should have been placed from the center of the room. 


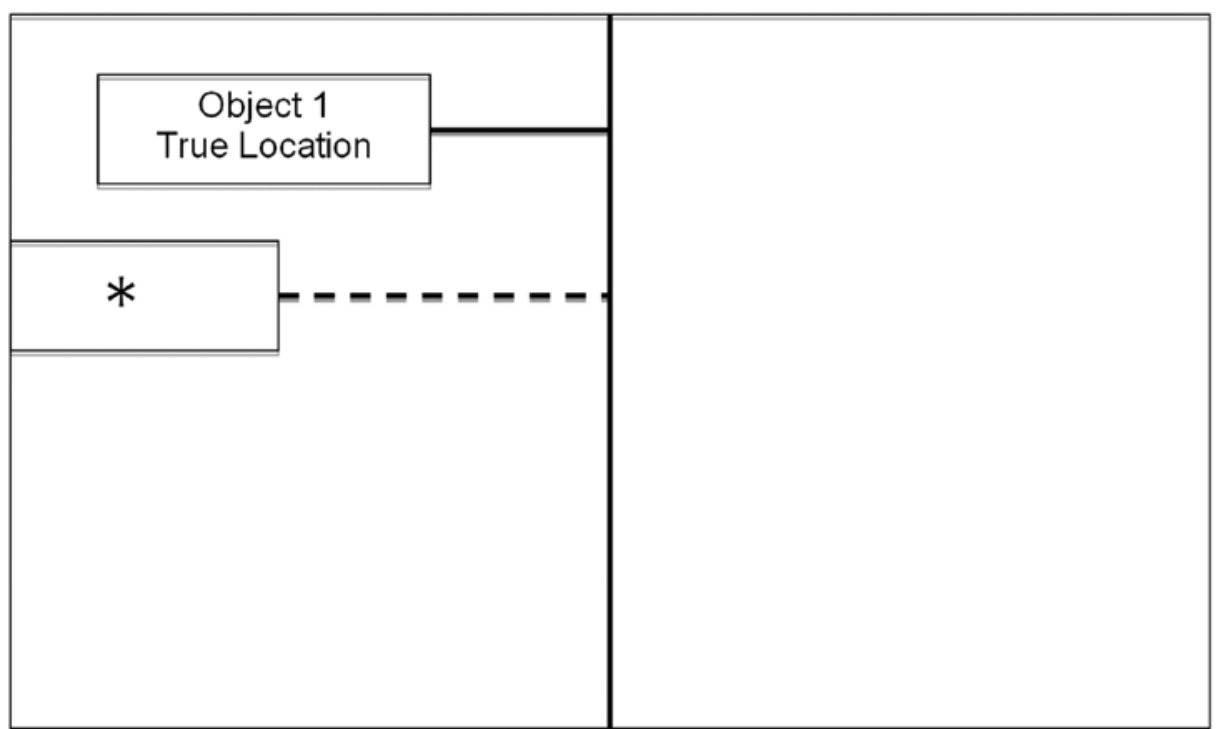

Figure 6. Sample of results with respect to vertical midline. * indicates participant's placement of object X. As can be seen here, by comparing the solid black line to the dotted black line, the response distance from the vertical midline is too large.

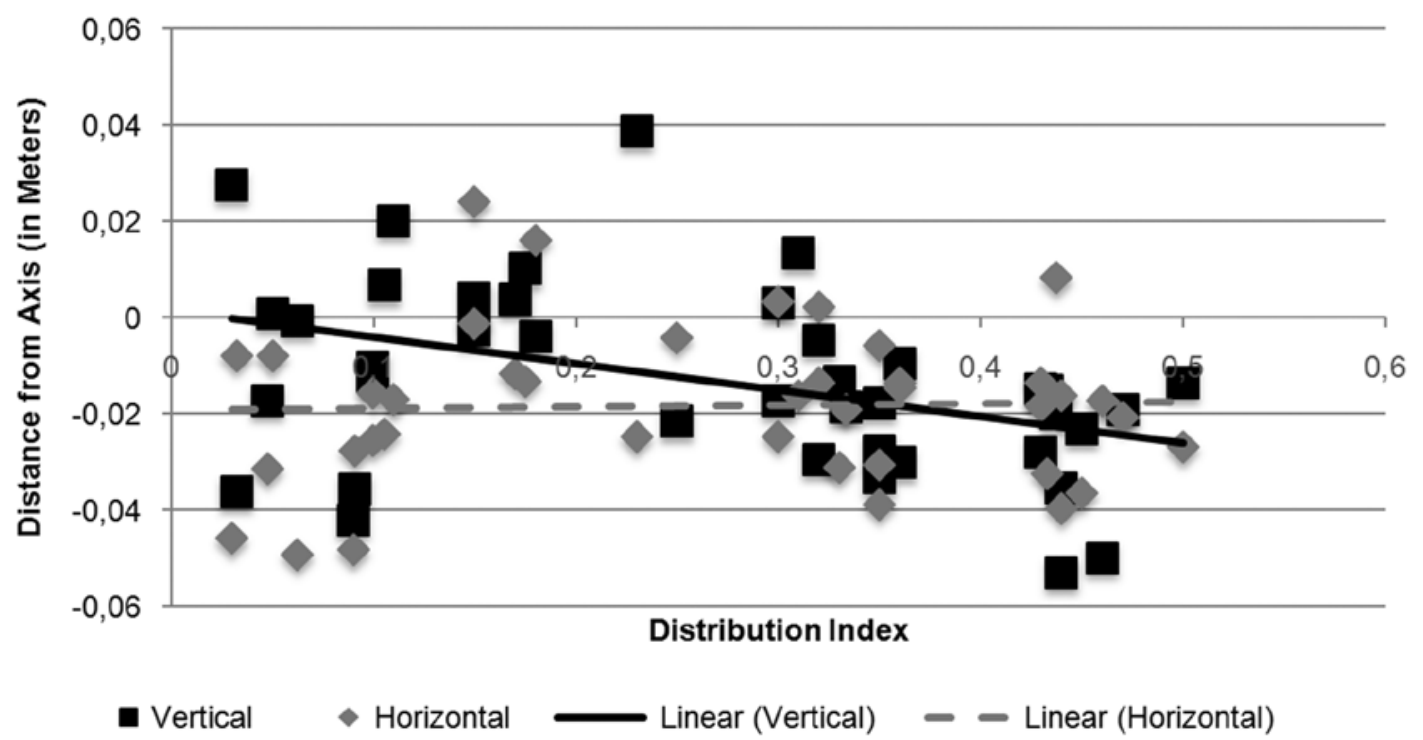

Figure 7. Relationship between distribution index and distance from the vertical (black squares) and horizontal (gray diamonds) midlines. As the distribution index increased, the distance from the vertical midline became increasingly negative, showing that on average objects were placed further than they should have been placed from the vertical midline. At the same time, no relationship existed between the distribution index and distance from the horizontal midline.

the vertical midline, with one group composed of objects to the left of the midline, in Half 1 (Figure 2; objects 1,5, 6 , and 7) and a second group composed of objects to the right of the midline, in Half 2 (Figure 2; objects 3 and 4). Object 2 was excluded as it fell directly on the vertical midline. For each participant, the average signed error in distance from the vertical midline was computed for the objects in the participants' preferred and non-preferred halves. An aggregate mislocation index was then created for each participant by taking the difference between the error for the non-preferred and preferred halves. This aggregate mislocation index was significantly predicted by the distribution index $(\beta=-193.26, \mathrm{t}=-2.18, \mathrm{p}=.04)$.
To further examine the relationship between the aggregate mislocation index and the distribution index, participants were split into two groups, based on whether their distribution index was below or above the mean value of the index across participants. Examination of signed error in distance from the vertical midline for objects in the preferred and non-preferred halves revealed distinct patterns in the two groups. The average value of the aggregate mislocation index was positive for participants with low values on the distribution index (who did not distribute views equally over the two available sides). These participants showed positive mislocation of objects in the non-preferred half 
$($ Mean $=2.63, \mathrm{SE}=6.6)$ and negative mislocation of objects in the preferred half $($ Mean $=-2.18, \mathrm{SE}=-9.78)$. In short, these participants mislocated objects toward their preferred side of the room (Figure 8). On the other hand, the average value of the aggregate mislocation index was negative for participants with high values on the distribution index (who did distribute views equally over the two available sides). These participants showed negative mislocation of objects in the non-preferred half (Mean $=-28.8, \mathrm{SE}=10.28)$ and negative mislocation of objects in the preferred half (Mean $=-10.77, \mathrm{SE}=6.12$ ). In short, these participants mislocated objects in each half of the room toward the corresponding side of the room (Figure 8).

To this point, our analyses have considered the sides from which participants viewed the room but not the corners within those sides. In a further analysis we considered the corners from which participants viewed the room. If the corners from which participants viewed the room were important in determining their memory for the room, we might expect that, to the degree that participants experienced the room along one diagonal, their error in locating objects in the room could be predicted in terms of relationships between the objects and this dominant diagonal. To examine this possibility, we constructed an index of diagonal dominance for each participant. Each time a participant viewed the room from either corner A or C (Figure 2) a value of 1 was assigned, and each time a participant viewed the room from either corner B or D a value of 2 was assigned. These values were then averaged to create a diagonal dominance index with a value between 1 and 2, with values closer to 1 indicating more views along the diagonal connecting corners $\mathrm{A}$ and $\mathrm{C}$ and values closer to 2 indicating more views along the diagonal connecting corners $\mathrm{B}$ and $\mathrm{D}$. To determine whether diagonal dominance predicted performance in the memory task, participants' signed error along each diagonal was determined. For each diagonal, one corner was defined as zero (in diagonal 1 , corner A and in diagonal 2, corner B). The signed error in distance along the diagonal was the difference between the correct distance along the diagonal and the participant's response distance along the diagonal. The diagonal dominance index did not predict signed error along either diagonal (A \& $\mathrm{C}$ diagonal: $\beta=-40.16, \mathrm{t}=$ $-.78, \mathrm{p}=.44 ; \mathrm{B} \& \mathrm{D}$ diagonal: $\beta=-33.61, \mathrm{t}=-.84, \mathrm{p}=$ $.41)$. This suggests that participants' error in mislocating the objects was not related to their experience of the room in terms of the diagonals. To further make the case that there was no relationship between diagonal dominance and error along the diagonal, we examined only participants with low values for the distribution index. As was previously shown, participants with low values for the distribution index mislocated objects along the horizontal axis towards their preferred side of the room. With this in mind, we would expect any relationship between diagonal dominance and error along the diagonals to be more pronounced among these participants. However, the diagonal dominance analysis remained non-significant even if limited to participants with lower values of the distribution index (A \& $\mathrm{C}$ diagonal: $\beta=-42.61, \mathrm{t}=-.81, \mathrm{p}=.42 ; \mathrm{B} \& \mathrm{D}$ diagonal: $\beta=-32.6, \mathrm{t}=-.76, \mathrm{p}=.45$ )

Finally we sought to model the process underlying our results. We considered the following simple account of these results: When a participant viewed an object from

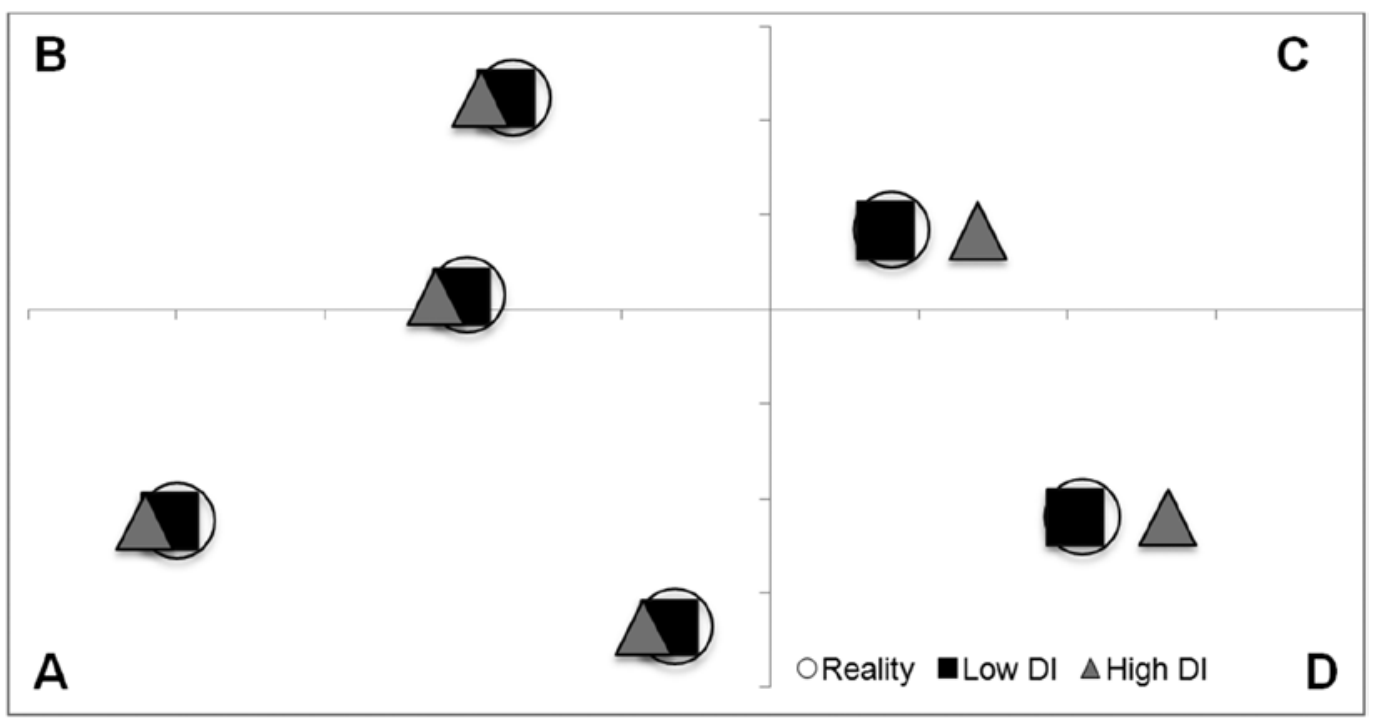

Figure 8. Average horizontal mislocation for objects on preferred and non-preferred side (with preferred side defined as corners A and B) for low and high distribution indices. Actual locations of objects are shown as empty circles. Average horizontal mislocations of objects for participants with below average distribution index are shown as black squares. Average horizontal mislocations of objects for participants with above average distribution index are shown as gray triangles. Participants who did not distribute views equally over the two available sides (low distribution index) had a positive aggregate mislocation index, reflecting mislocation towards their preferred side. Participants who did distribute views equally over the two available sides (high distribution index) had a negative aggregate mislocation index, reflecting mislocation of objects in each half of the room towards the corresponding side of the room. 
a given side of the room, the participant underestimated the distance between the side and the object, seeing the distance as a proportion of the true distance (we felt that the negative results of the diagonal analysis justified ignoring the specific corners from which participants viewed the room). The degree of underestimation differed for objects in the same half of the room as the participant's viewpoint and objects in the opposite half of the room. The effects of the underestimation were aggregated with an accumulator-like process (Heathcote \& Love, 2012) under which the location of all objects in the room was updated after each view of the room to reflect the underestimation occurring in that view. To the degree that the participant distributed his/her viewing unequally between Sides 1 or 2 , a constant biasing influence was applied in localization of the objects. As a result, all objects were mislocated toward the side from which the participant tended to see the room. To the degree that the participant distributed his/ her viewing equally between Sides 1 and 2, opposing biasing influences were applied in localization of the objects. Because the degree of underestimation differed for close and far objects, underestimation occurring from a given side tended to affect primarily objects in the corresponding half of the room. Thus, objects ended up being mislocated toward the sides to which they were close.

To test our account we fit the data for each participant by minimizing $D$, the summed horizontal distance between the correct and the remembered locations of the seven target objects. The two parameters of our Underestimated Distance model indexed the degree to which horizontal distances were underestimations between objects and the side of the room from which the room was viewed. Specifically, the parameters $f_{c}$ and $f_{f}$, indexed underestimated for objects that were in the same half of the room and in different halves of the room as the side in question. Both parameters varied between 0 and 1. Underestimation of distance occurred as follows: Starting with the actual horizontal distance $e_{1 j}$, from each object $j$ to Side 1, we reduced that distance each time the participant viewed the room from Side 1. Specifically, for objects in the half of the room corresponding to Side 1 , distance ${ }_{1 j}$ was set equal to $f_{c}$ distance $_{1 j}$. For objects in the opposite half of the room, distance ${ }_{l j}$ was set equal to $f_{f}$ distance $_{1 j}$. Each time the participant viewed the room from Side 2, we derived the horizontal distance 2 , from each object $j$ to Side 2 by taking the difference between the horizontal length of the room and the current

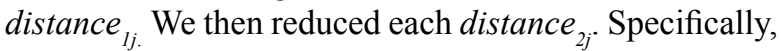
for objects in the half of the room corresponding to Side 2 , distance ${ }_{2 j}$ was set equal to $f_{c}$ distance $_{2 j}$. For objects in the opposite half of the room, distance ${ }_{2 j}$ was set equal to $f_{f}$ distance $_{2 j}$. We then derived each distance ${ }_{1 j}$ by taking the difference between the horizontal length of the room and each distance ${ }_{2 j}$. The Underestimated Distance model explained an average of $73 \%$ of the variance in the data for the individual participants. The amount of variance explained was statistically significant in the data for 28 of the 40 participants. The Underestimated Distance model can of course only be strictly evaluated in the context of competing models, and the present results do not provide an adequate foundation for such an evaluation. Nonetheless, these results suggest that the model may be worthy of further consideration.

\section{Discussion}

The present study examined memory for a domain after knowledge of the domain was acquired from two opposing points of view. Participants completed a search task in a 3-D model of a room, during which they were given the option of viewing the room from opposing sides. Following this search task, participants were asked to demonstrate their recall for the locations of the objects in the room by completing a reconstruction task. Subsequently, the relationship between performance in the search task and the reconstruction task was examined.

First we consider the results of the search task. We saw an effect of viewpoint distribution on search task performance such that equal distribution of views across both sides decreased correct responses regarding the location of the target. Our results do not speak to why equal distribution might have negatively impacted the search task, but this effect might be similar to the effect that Wang \& Simons (1999) observed, in which participants had a more difficult time detecting change when the display moved relative to them as compared to when they moved relative to the display. Wang \& Simons (1999) concluded that performance might have been reduced because moving the display did not allow for any updating based on body motion information (i.e., motor output and proprioceptive information). It is possible that a similar effect was at work here because participants did not physically move when alternating between different viewpoints. That is, as participants viewed the room from more disparate viewpoints, they were not as able to update their representations of the display and thus not as able to integrate the different views that they saw of the display.

Performance in the reconstruction task was also influenced by performance in the search task. As the distribution of participants' search viewpoints approached equivalence, participants demonstrated an increasingly negative radial signed error in the reconstruction task. That is, as the distribution approached equivalence, we saw a dispersive effect, such that participants placed objects increasingly too far from the center of the room as compared to the correct location. When error was further decomposed relative to the vertical and horizontal midlines, we found that the dispersive effect was driven by object placement with respect to the vertical midline. Specifically, as the distribution approached equivalence, participants placed objects too far from the vertical midline.

We compared the signed error in distance from the vertical midline for objects that were located in participants' preferred and non-preferred halves of the 
room. Participants who did not distribute views equally over the two available sides showed positive mislocation of objects in the non-preferred half and negative mislocation of objects in the preferred half. Thus, these participants mislocated objects toward their preferred side of the room. On the other hand, participants who did distribute views equally over the two available sides showed negative mislocation of objects in the nonpreferred and preferred halves. Thus, these participants mislocated objects in each half of the room toward the corresponding side of the room.

Taken together, these findings suggest that participants underestimated the distance between the objects and the sides of the room. Previous research has shown that exocentric distances are underestimated along the depth dimension (Loomis et al., 1992; Wu et al., 2008). In the current study, the manifestation of participants' underestimation of distance apparently depended on the participants' distribution of viewpoints. Participants who did not distribute views equally over the two available sides tended to remember the objects relative to a single side, rather than both sides. Underestimation of object distances from that preferred side resulted in mislocation of objects toward that side. Participants who did distribute views equally over the two available sides tended to remember the objects relative to both sides. Underestimation of object distances from the two sides resulted in mislocation of objects in each half of the room toward the corresponding side of the room.

We suggest that these findings are due to errors in the spatial representations formed by participants' during the search task. However, one possible limitation could be that errors arose from the spatial transformation required when changing from the perspective of the computer-generated room to the perspective of the reconstruction map. More specifically, participants may have used a ground-plane-based frame of reference during the search task, which they were then required to transform into more of a bird's eye frame of reference for the reconstruction map. We argue that this transformation is unlikely to have significantly altered the results because the viewpoint we presented during the search task was not truly ground-plane-based. The view presented (as seen in Figure 1) was not consistent with the participants standing directly on the ground, and in fact such a view would likely have made the search task impossible due to occlusion from the closest objects. The view participants were exposed to was actually closer to being a bird's eye view; thus, any transformation was likely very small.

The present research suggests one type of inaccuracy that occurs when participants attempt to integrate multiple views of a domain from opposing viewpoints. One key limitation in this study is that in presenting the participants with a computer-generated room, we significantly limited their access to typical depth cues. Specifically, participants' main depth cue was occlusion. By extension, it is possible that participants were limited to an ordinal metric in creating their reconstruction maps. The results that we discuss may be specific to this type of situation; therefore, it would be useful in the future to examine if the same effects occur in a more natural scene, with more varied depth cues available.

\section{References}

Castelhano, M. S., Pollatsek, A., \& Rayner, K. (2009). Integration of multiple views of scenes. Attention, Perception, \& Psychophysics, 71(3), 490-502. doi:10.3758/APP.71.3.490

Cherry, K. E., \& Park, D. C. (1993). Individual difference and contextual variables influence spatial memory in younger and older adults. Psychology and Aging, 8, 517- 526.

Diwadkar, V. A., \& McNamara, T. P. (1997). Viewpoint dependence in scene recognition. Psychological Science, 8, 302-307.

Edelman, S. (1999). Representation and recognition in vision. Cambridge, MA: MIT Press.

Friedman, A., \& Waller, D. (2008). View combination in scene recognition. Memory \& Cognition, 36, 467-478. doi:10.3758/ MC.36.3.467

Friedman, A., Waller, D., Thrash, T., Greenauer, N., \& Hodgson, E. (2011). View combination: a generalization mechanism for visual recognition. Cognition, 119, 229-241. doi:10.1016/j. cognition. 2011.01.012.

Gogel, W. C. (1965). Equidistance tendency and its consequences. Psychological Bulletin, 64, 153-163.

Heathcote, A., \& Love, J. (2012). Linear deterministic accumulator models of simple choice. Frontiers in Psychology, ArtID 292.

Hubbard, T. L., \& Ruppel, S. E. (2000). Spatial memory averaging, the landmark attraction effect, and representational gravity. Psychological Research, 64, 41-55.

Laurance, H. E., Thomas, K. G. F., Newman, M. C., Kaszniak, A. W., Nadel, L., Jacobs, W. J. (2002). Older adults map novel environments but do not place learn: findings from a computerized spatial task. Aging Neuropsychology and Cognition, 9(2), 85-97.

Loomis, J. M., DaSilva, J. A., Fujita, N., \& Fukusima, S. S. (1992). Visual space perception and visually directed action. Journal of Experimental Psychology: Human Perception and Performance, 18(4), 906-921.

Mou, W., Fan, Y., McNamara, T. P., \& Owen, C. (2008). Intrinsic frames of reference and egocentric viewpoints in scene recognition. Cognition, 106(2), 750-769. doi:10.1016/j.cognition.2007.04.009

Mou, W., Zhang, H., \& McNamara, T. P. (2009). Novel-view scene recognition relies on identifying spatial reference directions. Cognition, 111(2), 175-186. doi:10.1016/j. cognition. 2009.01.007

Tarr, M. J. (1995). Rotating objects to recognize them: a case study on the role of viewpoint dependency in the recognition of threedimensional objects. Psychonomic Bulletin \& Review, 2, 55-82.

Ullman, S. (1998). Three-dimensional object recognition based on the combination of views. Cognition, 67(1-2), 21-44.

Wang, R. F., \& Simons, D. J. (1999). Active and passive scene recognition across views. Cognition, 70(2), 191-210.

Wu, J., He, Z. J., \& Ooi, T. L. (2008). Perceived relative distance on the ground affected by the selection of depth information. Perception \& Psychophysics, 70(4), 707-713. doi:10.3758/PP.70.4.707. 